$02,11,12$

\title{
Влияние локальных корреляций на переход „однородный изолятор-сверхпроводник“ в доменных границах фазы зарядового порядка 2D-системы со смешанной валентностью
}

\author{
(C) В.В. Конев, В.А. Улитко, Д.Н. Ясинская, Ю.Д. Панов, А.С. Москвин
}

Уральский фредеральный университет,

Екатеринбург, Россия

E-mail: vitaliy.konev@urfu.ru

\begin{abstract}
В рамках (псевдо)спинового $S=1$ формализма показано, что структура антифазных доменных границ в фазе зарядового упорядочения системы со смешанной валентностью типа „триплета“ $\mathrm{Cu}^{1+, 2+, 3+}$ в купратах на двумерной квадратной решетке существенно зависит от параметра одноцентровых корреляций $U$. Компьютерное моделирование на больших квадратных решетках иллюстрирует изменение структуры границ от однородной моновалентной непроводящей типа $\mathrm{Cu}^{2+}$ до нитевидной сверхпроводящей при относительно небольшом изменении положительных значений $U$.
\end{abstract}

Работа выполнена при поддержке Программы 211 Правительства Российской Федерации, соглашение № 02.А03.21.0006 и проектов № 2277 и № 5719 Министерства Образования и Науки Российской Федерации.

DOI: 10.21883/FTT.2018.11.46644.01NN

\section{1. Введение}

Наряду с сильноанизотропными магнетиками на основе $\mathrm{Ni}^{2+}(S=1)$, например, $\left.\left[\mathrm{Ni}\left(\mathrm{HF}_{2}\right)(3-\mathrm{Clpy})_{1}\right] \mathrm{BF}_{1}\right]$ и $\mathrm{NiCl}_{2} 4 \mathrm{SC}\left(\mathrm{NH}_{2}\right)_{2}$, интерес к системам со спином $S=1$ связан и с так называемыми псевдоспиновыми системами типа „полужестких“ (semi-hard-core) бозонов с ограничением на заполнение узлов решетки $n=0,1,2$, или системами ионов со смешанной валентностью типа „триплета“ $\mathrm{Cu}^{1+, 2+, 3+}$ в купратах $\mathrm{La}_{2-x} \mathrm{Sr}_{x} \mathrm{CuO}_{4}, \mathrm{Bi}^{3+, 4+, 5+}$ в висмутатах [1]. Во всех случаях фазованя диаграмма спиновых или псеводоспиновых систем с $(S=1)$ существенно богаче, чем в случае аналогичных систем с квантовым (псевдо)спином $S=1 / 2$, прежде всего за счет появления принципиально новых слагаемых в гамильтониане типа одноионной анизотропии и биквадратичных взаимодействий и принципиально новых фаз типа квантового парамагнетика и спин-нематика.

В зависимости от величины параметров локальных и межцентровых заряд-зарядовых корреляций, интегралов одно- и двухчастичного переноса, а также полного заряда, основное состояние таких систем может соответствовать зарядовому упорядочению, различным типам сверхпроводящего упорядочения, комбинированным фазам типа „суперсолида“ (supersolid) с сосуществованием сверхпроводимости и зарядового порядка, а также специфической для этих систем фазе типа квантового парамагнетика. Для этих систем характерно формирование различных метастабильных неоднородных состояний с развитой доменной структурой и топологическими дефектами типа вихрей и скирмионов [2-4].

В данной работе в рамках псевдоспинового формализма рассмотрена простая система зарядовых триплетов $\mathrm{Cu}^{1+, 2+, 3+}$ в модельном купрате. Мы показываем, что структура антифазных доменных границ в фазе зарядового упорядочения такой системы существенно зависит от параметра одноцентровых корреляций $U$, меняясь от однородной моновалентной непроводящей типа $\mathrm{Cu}^{2+}$ до нитевидной сверхпроводящей при относительно небольшом изменении положительных значений $U$.

\section{2. Модельный купрат: псевдоспиновый $S=1$ формализм}

Модельный купрат представляет 2D-систему Cu-центров в $\mathrm{CuO}_{2}$ плоскости купратов, которые могут находиться в трех различных валентных зарядовых состояниях: $\mathrm{Cu}^{1+, 2+, 3+}$. Этот зарядовый триплет мы связываем с тремя состояниями псевдоспина $S=1$ следующим образом: $\mathrm{Cu}^{1+} \rightarrow M_{S}=-1, \mathrm{Cu}^{2+} \rightarrow M_{S}=0$, $\mathrm{Cu}^{3+} \rightarrow M_{S}=+1$ и используем известные методы описания спиновых систем.

Спиновая алгебра систем с $S=1\left(M_{S}=0, \pm 1\right)$ включает восемь независимых нетривиальных операторов (три дипольных и пять квадрупольных)

$$
\begin{gathered}
S_{z} ; S_{ \pm}=\mp \frac{1}{\sqrt{2}}\left(S_{x} \pm i S_{y}\right) ; S_{z}^{2} ; \\
T_{ \pm}=\left\{S_{z}, S_{ \pm}\right\} \equiv S_{z} S_{ \pm}+S_{ \pm} S_{z} ; S_{ \pm}^{2} .
\end{gathered}
$$

Операторы повышения/понижения $S_{ \pm}$и $T_{ \pm}$меняют проекцию псевдоспина на \pm 1 , но различным образом: $\left\langle 0\left|S_{ \pm}\right| \mp 1\right\rangle=\left\langle \pm 1\left|S_{ \pm}\right| 0\right\rangle=\mp 1, \quad\left\langle 0\left|T_{ \pm}\right|=\mp 1\right\rangle$ $=-\left\langle \pm 1\left|T_{ \pm}\right| 0\right\rangle=+1$. Операторы повышения/понижения $S_{ \pm}^{2}$ описывают переходы $|-1\rangle \leftrightarrow|+1\rangle$, то есть они „рождают“ на узле дырочную $\left(S_{+}^{2}\right)$ или электронную $\left(S_{-}^{2}\right)$ пару, представляющих композитный локальный бозон, с кинематическим ограничением $\left(S_{ \pm}^{2}\right)^{2}=0$, что подчеркивает его природу как „жесткого“ (hard-core) 
бозона. Локальный (узельный) недиагональный параметр порядка $\left\langle S_{ \pm}^{2}\right\rangle$, являющийся фактически параметром локального сверхпроводящего порядка, отличен от нуля только в случае, если на узле имеется квантовая суперпозиция состояний $|-1\rangle$ и $|+1\rangle$.

Вводя псевдоспиновый $S=1$ формализм для описания зарядовых триплетов, запишем эффективный гамильтониан, который коммутирует с $z$-компонентой полного псевдоспина $\Sigma_{i} S_{i z}$ и, таким образом, сохраняет полный заряд системы, как сумму потенциальной и кинетической энергий

$$
H=H_{\text {pot }}+H_{\text {kin }}^{(2)}
$$

где

$$
H_{\mathrm{pot}}=\sum_{i}\left(\Delta S_{i z}^{2}-\mu S_{i z}\right)+\frac{1}{2} V \sum_{\langle i j\rangle} S_{i z} S_{j z},
$$

а в кинетической энергии мы учтем только вклад двухчастичного переноса локальных композитных бозонов

$$
H_{\mathrm{kin}}^{(2)}=-\frac{1}{2} t_{b} \sum_{\langle i j\rangle}\left(S_{i+}^{2} S_{j-}^{2}+S_{i-}^{2} S_{j+}^{2}\right) .
$$

Первое слагаемое в (3), или „одноионная анизотропия“, описывает корреляционные эффекты плотностьплотность на узлах, причем параметр $\Delta$ можно связать с известным корреляционным параметром $U: \Delta=U / 2$. Второе слагаемое может быть связано с псевдомагнитным полем вдоль оси $O z$, либо с химическим потенциалом относительно добавления новых частиц. Последний член описывает межузельные взаимодействия (корреляции) типа плотность-плотность. Ниже мы ограничиваемся учетом взаимодействия ближайших соседей с положительным (антиферромагнитным) знаком параметра межцентровых корреляций $V$.

В зависимости от соотношения между параметрами гамильтониана (2) и величины полного заряда основное состояние системы соответствует либо однородной непроводящей фазе типа квантового парамагнетика с $\left\langle S_{z}\right\rangle=\left\langle S_{z}^{2}\right\rangle=0$, реализуемой при больших положительных значениях корреляционного параметра $\Delta$ (large- $U$ phase), либо непроводящей фазе зарядового упорядочения (CO) - аналогу антиферромагнитного упорядочения вдоль $z$-оси, либо сверхпроводящей (SF, superfluid) фазе с отличным от нуля параметром порядка $\left\langle S_{ \pm}^{2}\right\rangle$, сопровождаемой однородным ферроупорядочением, или неоднородным антиферроупорядочением (supersolid phase) z-компонент псевдоспина. Локальный параметр сверхпроводящего порядка $\left\langle S_{ \pm}^{2}\right\rangle$ может быть представлен в стандартной форме $|\Psi| e^{ \pm i \phi}$ с модулем $|\Psi|$ и фазой $\phi$.

\section{3. Особенности структуры антифазных доменных границ СО-фазы}

С использованием графического процессора NVidia в рамках метода Монте-Карло мы моделировали фазовый
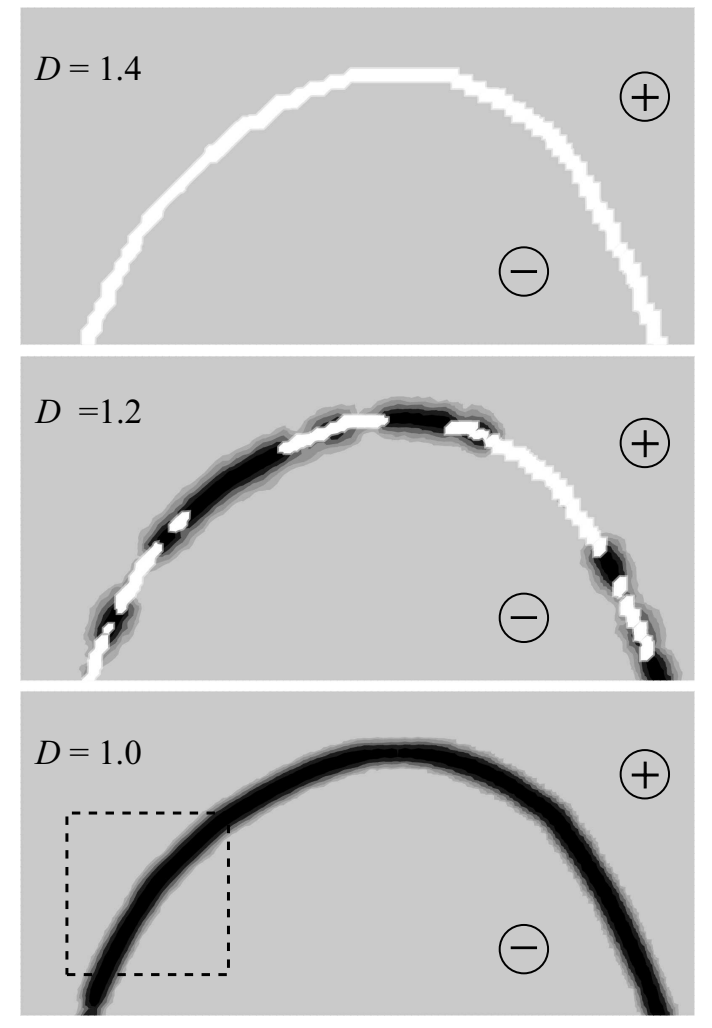

Рис. 1. Эволюция антифазной доменной границы с ростом параметра локальной корреляции $\Delta$. Выделен фрагмент решетки $256 \times 256$ с антифазной доменной границей, разделяющей домены зарядового порядка, обозначенные знаками плюс и минус. Черным ибелым цветом обозначены нитевидные „сверхпроводящая“ и „родительская“ $\mathrm{Cu}^{2+}$-фазы соответственно.

переход зарядового упорядочения в модельном купрате в двух-подрешеточном приближении для квадратной решетки $256 \times 256$ с периодическими граничными условиями при выборе параметров $t_{b}=1, V=0.75, \mu=0$, обеспечивающим основное состояние типа зарядового упорядочения в достаточно широком диапазоне изменений параметра локальной корреляции $\Delta$.

В процессе быстрой термализации (отжига), при $\Delta=-5$, происходило формирование разветвленной доменной структуры с появлением при низких температурах ярко выраженной нитевидной (филаментарной) сверхпроводимости в центре антифазных доменных границ СО-фазы, характеризуемой, прежде всего, отличным от нуля значением модуля локального параметра сверхпроводящего порядка, свидетельствующего о существовании локальных квантовых суперпозиций $\mathrm{Cu}^{1+}-\mathrm{Cu}^{3+}$. C ростом интеграла переноса композитного бозона $t_{b}$ происходит постепенное уширение доменных границ с увеличением объема сверхпроводящего состояния вплоть до полного вытеснения СО-фазы и перехода в неоднородное сверхпроводящее состояние.

Интересно, что как СО-доменная структура, так и сверхпроводящая структура доменной границы оказа- 


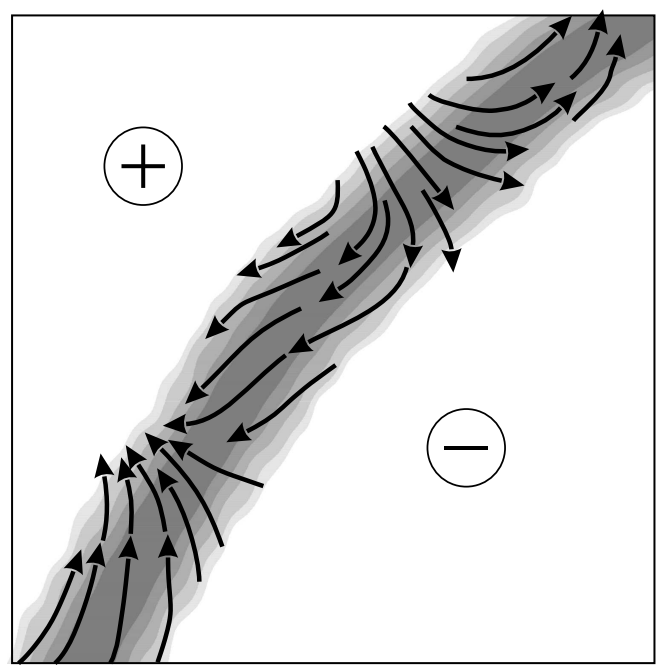

Рис. 2. Распределение фазы локального сверхпроводящего параметра порядка (фазовый поток) в участке доменной границы, выделенном рамкой на рис. 1. Градация серого цвета указывает на неоднородность распределения модуля локального параметра сверхпроводящего порядка.

лись устойчивыми относительно изменения параметра $\Delta$ локальной корреляции в широком диапазоне, вплоть до значений $\Delta \approx+1.0$. Однако при дальнейшем росте локальных корреляций происходит коренная перестройка структуры доменных границ. На рис. 1 представлена картина эволюции антифазной доменной границы с ростом $\Delta \geq+1.0$, а на рис. 2 представлено распределение фазы локального сверхпроводящего параметра порядка (фазовый поток) в доменной границе. При постепенном повышении $\Delta$ нарушается регулярная структура нитевидной сверхпроводимости в центре антифазной доменной границы с появлением участков „родительской“ $\mathrm{Cu}^{2+}$-фазы, то есть фазы квантового парамагнетика на псевдоспиновом языке, разрастающейся вплоть до полного вытеснения нитевидной сверхпроводимости при $\Delta \approx+1.4$ и перехода всей границы в $\mathrm{Cu}^{2+}$-фазу. При дальнейшем росте корреляций $\Delta \geq+1.5$ происходит уширение доменной границы с постепенным вытеснением зарядового порядка. Другими словами, фазовый переход: зарядовое упорядочение родительская фаза (large- $U$-phase) с ростом параметра локальной корреляции реализуется путем разрастания доменных границ.

Исследование температурных эффектов показывает, что с ростом температуры в доменных стенках CO-фазы при $\Delta=+1.0$ сперва происходит переход из сверхпроводящего состояния в родительскую фазу, а затем в неупорядоченное „парамагнитное“ состояние. Однако при последующем охлаждении вплоть до очень низких температур $T=0.0001$ восстанавливается только „родительская“ структура доменных границ, то есть мы имеем дело с температурным гистерезисом структуры границ.

\section{4. Заключение}

Нами проведено исследование влияния величины локальных корреляций $\Delta=U / 2$ на структуру доменных границ фазы зарядового упорядочения модельного купрата. В ходе численного моделирования методом МонтеКарло на больших квадратных решетках мы смогли наблюдать образование при быстром отжиге развлетвленной доменной структуры, в антифазных доменных границах которой формируется нитевидная филаментарная сверхпроводимость, устойчивая в широком интервале изменений $U$ вплоть до положительных значений $U \approx 2$. Однако при дальнейшем росте локальных корреляций нитевидная сверхпроводимость разрушалась и в границах формировалась нитевидная „родительская“ $\mathrm{Cu}^{2+}$-фаза, разделяющая домены с зарядовым упорядочением $\mathrm{Cu}^{1+}-\mathrm{Cu}^{3+}$. Моделирование температурных эффектов указывает на наличие температурного гистерезиса структуры границ.

\section{Список литературы}

[1] A.S. Moskvin. ЖЭТФ 121, 3, 477 (2015); [JETP 121, 3, 549 (2015)].

[2] Y.D. Panov, A.S. Moskvin, F.N. Rybakov, A.B. Borisov. J. Low Temperature Phys. 185, 5-6, 488 (2016).

[3] A.S. Moskvin, Yu.D. Panov, F.N. Rybakov, A.B. Borisov. J. Superconductiv. Nov. Magnet. 30, 1, 43 (2017).

[4] Y.D. Panov, A.S. Moskvin. Physica C 548, 82 (2018); https://doi.org/10.1016/j.physc.2018.02.032.

Редактор Т.Н. Василевская 